\title{
Whole Genome Sequence of Multiple Myeloma-Prone C57BL/KaLwRij Mouse Strain Suggests the Origin of Disease Involves Multiple Cell Types
}

Sarah R. Amend1, William C. Wilson 1, Liang Chu', Lan Lu1, Pengyuan Liu², Daniel Serie ${ }^{5}$, Xinming Su${ }^{1}$, Yalin $\mathrm{Xu}^{1}$, Dingyan Wang ${ }^{4}$, Anthony Gramolini ${ }^{4}$, Xiao-Yan Wen ${ }^{4}$, Julie O'Neal ${ }^{1}$, Michelle Hurchla ${ }^{1}$, Celine M. Vachon ${ }^{5}$, Graham Colditz ${ }^{3}$, Ravi Vij ${ }^{1}$, Katherine N. Weilbaecher ${ }^{1}$, Michael H. Tomasson ${ }^{1}{ }^{*}$

1 Division of Oncology, Washington University School of Medicine, St. Louis, MO, United States of America, 2 Medical College of Wisconsin, Milwaukee, WI, United States of America, 3 Department of Surgery, Washington University School of Medicine, St. Louis, MO, United States of America, 4 Department of Physiology, University of Toronto, Toronto, Canada, 5 Department of Health Sciences Research, Division of Epidemiology, Mayo Clinic College of Medicine, Rochester, MN, United States of America

* tomasson@dom.wustl.edu

Citation: Amend SR, Wilson WC, Chu L, Lu L, Liu P, Serie D, et al. (2015) Whole Genome Sequence of Multiple Myeloma-Prone C57BL/KaLwRij Mouse Strain Suggests the Origin of Disease Involves Multiple Cell Types. PLOS ONE 10(5): e0127828. doi:10.1371/journal.pone.0127828

Academic Editor: Claire M. Edwards, University of Oxford, UNITED KINGDOM

Received: February 2, 2015

Accepted: March 10, 2015

Published: May 28, 2015

Copyright: @ 2015 Amend et al. This is an open access article distributed under the terms of the Creative Commons Attribution License, which permits unrestricted use, distribution, and reproduction in any medium, provided the original author and source are credited.

Data Availability Statement: All relevant data are within the paper and it's supporting information files. The KaLwRij whole genome sequence are deposited in GenBank under accession number SRP057008.

Funding: This work was supported by the National Institute on Aging R21AG040777 (to MHT), the National Cancer Institute 1F31CA174096-01A1 (to SRA), the National Cancer Institute R01 CA097250, 2P01 CA100730 (to KNW, MH, YX, SRA, and XS), the St. Louis Men's Group against Cancer and Barnes Jewish Foundation (to KNW, MH, YX, SRA, and $X S$ ), and the Washington University

\section{Abstract}

Monoclonal gammopathy of undetermined significance (MGUS) is the requisite precursor to multiple myeloma (MM), a malignancy of antibody-producing plasma B-cells. The genetic basis of MGUS and its progression to MM remains poorly understood. C57BL/KaLwRij (KaLwRij) is a spontaneously-derived inbred mouse strain with a high frequency of benign idiopathic paraproteinemia (BIP), a phenotype with similarities to MGUS including progression to MM. Using mouse haplotype analysis, human MM SNP array data, and whole exome and whole genome sequencing of KaLwRij mice, we identified novel KaLwRij gene variants, including deletion of Samsn1 and deleterious point mutations in Tnfrsf22 and Tnfrsf23. These variants significantly affected multiple cell types implicated in MM pathogenesis including B-cells, macrophages, and bone marrow stromal cells. These data demonstrate that multiple cell types contribute to MM development prior to the acquisition of somatic driver mutations in KaLwRij mice, and suggest that MM may an inherently non-cell autonomous malignancy.

\section{Introduction}

Multiple myeloma (MM) is the second most common hematological malignancy and is characterized by accumulation of clonal plasma B-cells in the bone marrow, hypercalcemia, renal failure, anemia, and lytic bone lesions. Despite impressive recent progress in treatments for MM, median survival is only 6 years [1]. The requisite precursor to MM is monoclonal gammopathy 
Musculoskeletal Research Center (National Institutes of Health P30 AR057235) (to MH). The funders had no role in the study design, data collection and analysis, decision to publish, or preparation of the manuscript.

Competing Interests: The authors have declared that no competing interests exist. of undetermined significance (MGUS), a pre-neoplastic proliferation of clonally derived plasma cells without end organ damage.

Epidemiological studies suggest a genetic component to MM disease risk that is due to an increase MGUS development. African Americans have an increased risk of MM due to elevated MGUS risk, rather than an increased rate of conversion from MGUS to overt MM [2-4]. Moreover, inherited risk variants for MM also confer a significant increase in risk for MGUS [5], providing further evidence that increased MM susceptibility is due to inherited predisposition to MGUS. Interestingly, the somatic mutations responsible for disease progression from MGUS to MM remain unknown. The majority of somatic mutations found in CD138-selected MM cells are present at similar frequencies in similar cells isolated from MGUS patients [6], suggesting that plasma-cell extrinsic factors may contribute to disease progression in MM.

Few experimental models exist to study the biology of MGUS and MM in a laboratory setting, confounding efforts to understand the biology of MM progression. Xenograft models to study human myeloma growth, e.g. SCID-hu, require the presence of a human bone marrow microenvironment [7], highlighting the importance of non-malignant cells within the myeloma microenvironment.

The C57BL/KaLwRij mouse (KaLwRij) is a spontaneously-derived inbred mouse strain identified nearly 40 years ago that is predisposed to myeloma [8]. KaLwRij mice develop benign idiopathic paraproteinemia (BIP), a condition analogous to human MGUS, at a high rate. Affected mice progress to myeloma at the same low rate as humans do, approximately $1 \%$ per year. The 5TGM1 cell line originally isolated from a sick KaLwRij mouse is often used as a model of myeloma because when transplanted back into KaLwRij mice, it causes disease with similar clinical features as human MM including lytic bone lesions [8]. Notably, while the 5TGM1/KaLwRij model recapitulates many features of human disease, the genetic basis of BIP susceptibility in KaLwRij mice remains unknown.

In this study, we investigated the genetic determinants underlying the BIP predisposition of the KaLwRij strain. Using an integrative genetics approach, including whole genome and exome sequencing, we identified novel KaLwRij gene variants, such as homozygous deletion of Samsn1 and deleterious point mutations in tumor necrosis factor receptor family members. KaLwRij genetic variants significantly affected multiple cell types implicated in MM pathogenesis including B-cells, macrophages, and bone marrow stromal cells. These results illuminate pathways responsible for MM disease risk, and demonstrate for the first time that the development of myeloma involves multiple cell types prior to the acquisition of somatic mutations.

\section{Results}

We mapped genetic distances among myeloma-prone KaLwRij and eleven diverse inbred mouse strains using SNP arrays. KaLwRij was most closely related to its parent strain C57BL/6 (Fig 1A). Initially we hypothesized that KaLwRij predisposition to BIP would be reflected in a unique antibody response to immune challenge and that sustained serum immunoglobulin levels would provide a measurable quantitative phenotype to perform quantitative trait loci (QTL) mapping. Following immunization of these twelve strains (S1A Fig), analysis of serial serum samples by immunoglobulin ELISA demonstrated that the antibody response was highly heritable $\left(\operatorname{IgG~} \mathrm{h}^{2}=0.7247, \operatorname{IgM~h}^{2}=0.9551, \operatorname{IgA~}^{2}=1.019\right)$, indicating influence by genetic background (S1B-S1D Fig). Serum protein electrophoresis (SPEP), a standard diagnostic test for human MGUS, was used to identify M-spikes indicative of BIP (S1E Fig). Most strains presented with an M-spike immediately following immunization, indicating a normal immune response (S1 Table). M-spike presentation may be due either to increased survival of plasma cells or increased activation of memory B-cells, but work beyond the scope of this manuscript is 
a

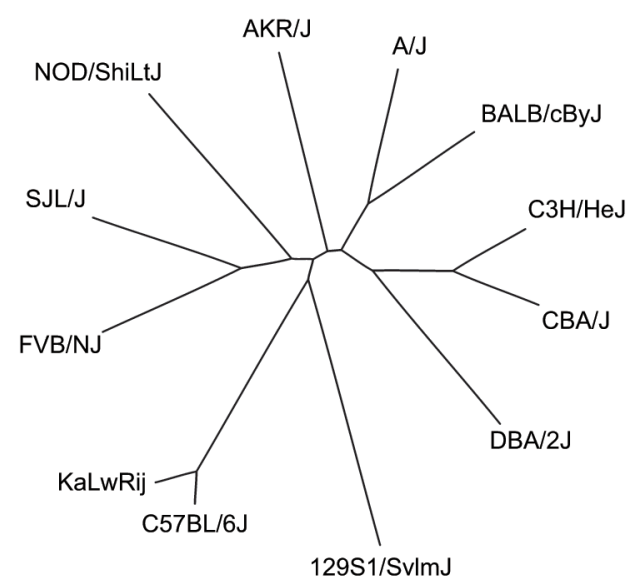

C

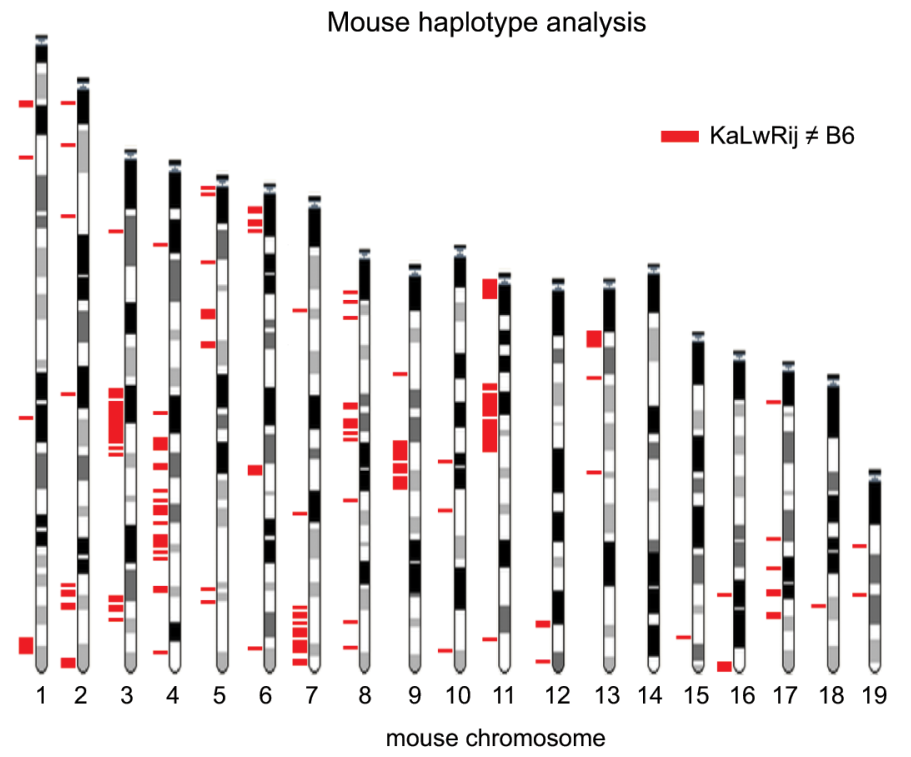

b

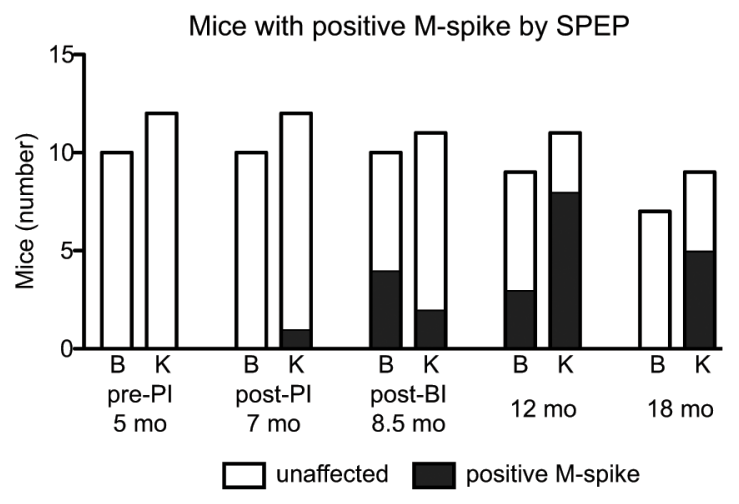

d

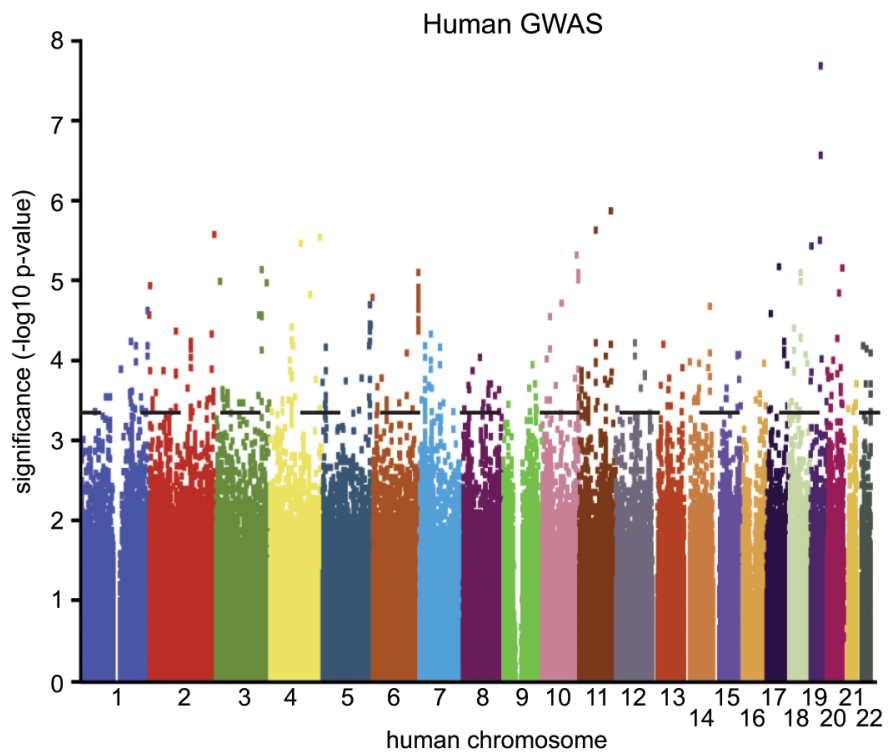

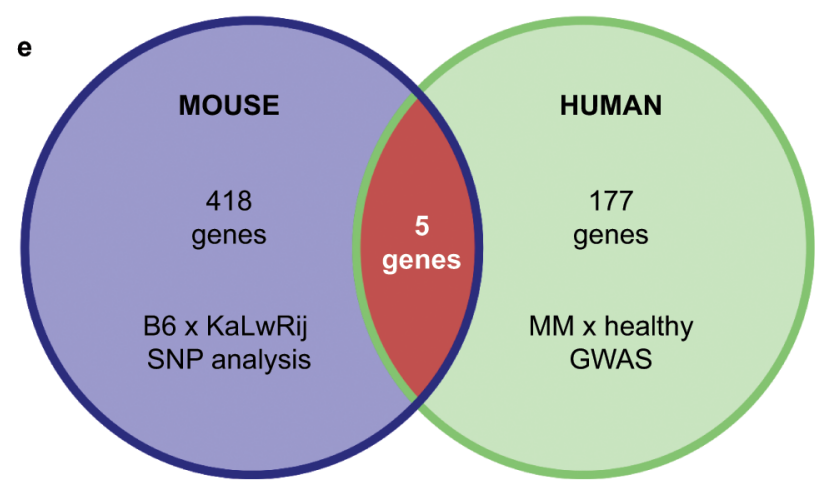

Fig 1. The KaLwRij strain was predisposed to BIP and intersecting mouse and human genetic analyses identified candidate genes that may influence murine BIP risk and human MM risk. (a) Phylogenetic tree demonstrating genetic distances of 12 inbred strains of mice. (b) Number of C57BL/6 and KaLwRij mice with positive M-spike by SPEP. (c) Haplotype analysis identified contiguous regions of non-shared polymorphic alleles between KaLwRij and C57BL/6 mice (red bars) in 419 genes. (D) GWAS between MM patients and healthy volunteers. SNPs in the 99th percentile (dashed line) fell in 178 genes. (e) Venn diagram representing combined analysis of (c) and (d), resulting in a candidate gene list of 5 genes.

doi:10.1371/journal.pone.0127828.g001 
necessary to dissect these possibilities. The highest frequency of an abnormal M-spike sustained to 18 months was found in KaLwRij (56\%) while it had resolved in C57BL/6 mice (Fig $1 B)$. The 18-month time frame and qualitative nature of the BIP phenotype prevented us from further pursuing QTL mapping.

We took advantage of the close genetic distance between BIP-resistant C57BL/6 and BIPsusceptible KaLwRij mouse strains to use haplotype mapping to identify BIP candidate genes. Of 562,061 single nucleotide polymorphisms (SNPs) queried, 21,133 SNPs varied between KaLwRij and C57BL/6 (3.76\%). A ranked list, defined by blocks of five or greater physically consecutive divergent SNPs, identified 418 candidate genes different between C57BL/6 and KaLwRij (Fig 1C, S2 Table). To enrich for candidate genes relevant to human MM, we took an integrative cross-species approach. We performed genome-wide association analysis (GWAS) on genomic DNA isolated from normal tissue of $305 \mathrm{MM}$ patients and 353 healthy controls to identify common genetic variants associated with MM. The relatively small patient population identified only one SNP (rs1029654 in an intergenic region) that reached genome-wide significance. To include additional genetic variants associated with MM risk, we queried SNPs in the 99th significance percentile (209 SNPs, Fig 1D) and generated a candidate gene list of 177 genes possibly influencing MM risk in humans (S3 Table). Importantly, this approach identified SNPs in three of the seven previously published genetic loci associated with MGUS and MM risk (2p23.3, 3p22.1, and 7p15.3), validating our approach. The intersection of the KaLwRij and C57BL/6 haplotype gene set (418 genes) and the human GWAS set (177 genes) contained five genes: Fstl4, Samsn1, Ccm2, Tenm3, and Csmd1 (Fig 1E).

To characterize these loci at base-pair resolution and to identify additional genomic variants contributing to MM pathogenesis, we performed whole genome sequencing (WGS) and whole exome sequencing (WES). 926,326,580 reads were obtained by WGS and 75,950,592 by WES, with $96.0 \%$ and $98.9 \%$ mapping to the reference C57BL/6 genome respectively. These data were analyzed for large deletions, single nucleotide variants (SNVs), and small insertion or deletion events (S4-S7 Tables). 19,042 cross-validated SNVs were identified in the KaLwRij genome (S5 Table and data not shown). Of these SNVs, 1,128 (5.9\%) resulted in nonsynonymous coding sequence changes (S5 Table), with 29 novel variants predicted to be disruptive (Table 1).

Analysis of structural variants identified two deletions. The first, confirmed by PCR and sequence analysis, spanned $1.6 \mathrm{~kb}$ within Rfpl3s (data not shown). More compelling, however, was a large $180 \mathrm{~kb}$ deletion encompassing the entire Samsn1 gene (Chr16: 7581619075996162; Fig 2A, S2A Fig), which was also identified in our cross-species approach.

To characterize the functional effect of Samsn1 deletion, we utilized a genetic mouse model with targeted deletion of Samsn1 on the C57BL/6 background. Samsn $1^{-/-}$mice have increased B-cell proliferation in vitro and increased immunoglobulin response in the weeks following immunization in vivo [9]. The $180 \mathrm{~kb}$ deletion resulted in an effective Samsn1 knock-out in KaLwRij mice, so we predicted KaLwRij mice would display similar B-cell phenotypes observed in Samsn $1^{-/-}$mice. Samsn1 was expressed in C57BL/6 but not in KaLwRij ex vivo stimulated splenic B-cells, confirming the loss of protein expression predicted by gene deletion (Fig 2B). $\mathrm{B}$-cells isolated from young KaLwRij prior to onset of BIP and myeloma had significantly increased proliferation following stimulation compared to C57BL/6 (Fig 2C). Additionally, KaLwRij mice had a significant and progressive elevation in immunoglobulin IgG2b levels following immunization (Fig 2D).

We next tested whether Samsn 1 expression played a role in fully transformed myeloma cells. The 5TGM1 myeloma cell line, originally isolated from a myeloma-bearing KaLwRij mouse [10], was confirmed as Samsn1-null (Fig 2E, S2A Fig). Stable re-expression of Samsn1 decreased proliferation of 5TGM1 cells compared to control under both basal and IL-6 
Table 1. KaLwRij novel germline missense, stoploss, and stopgain mutations.

\begin{tabular}{|c|c|c|c|c|}
\hline Chr & Position (bp) & Gene ID & Gene Name & Variant \\
\hline 2 & 20861102 & Arhgap21 & Rho GTPase activating protein 21 & c.C3215T:p.T1072I \\
\hline 3 & 95130195 & Scnm1 & Sodium channel modifier 1 & c.T559C:p.stop187R \\
\hline 4 & 88403392 & Focad & Focadhesin & c.A4979T:p.N1660I \\
\hline 7 & 140274118 & 5830411NO6Rik & Riken cDNA 5830411N06 gene & c.C1033T:p.Q345stop \\
\hline 7 & 140466879 & Olfr533 & Olfactory receptor protein 533 & c.G677A:p.R226H \\
\hline 7 & 140466884 & Olfr533 & Olfactory receptor protein 533 & c.C682T:p.R228C \\
\hline 7 & 140503539 & Olfr536 & Olfactory receptor protein 536 & c.C919T:p.R307C \\
\hline 7 & 140691588 & Olfr45 & Olfactory receptor protein 45 & c.C682T:p.R228C \\
\hline 7 & 143643365 & Tnfrsf22 & Tumor necrosis factor receptor superfamily, member 22 & c.A236C:p.Q79P \\
\hline 7 & 143680034 & Tnfrsf23 & Tumor necrosis factor receptor superfamily, member 23 & c.A206C:p.Q69P \\
\hline 8 & 53513574 & Aga & Aspartylglucosaminidase & c.C160A:p.L54M \\
\hline 11 & 3179430 & Sfi1 & Sfi1 homolog, spindle assembly associated (yeast) & c.G344A:p.W115stop \\
\hline 11 & 3923487 & Ten2 & Transcobalamin 2 & c.C858G:p.F286L \\
\hline 11 & 4075493 & $\operatorname{Sec} 14 / 3$ & SEC14-like 3 (S. cerevisiae) & c.C1016A:p.T339N \\
\hline 11 & 5144386 & Emid1 & EMI domain containing 1 & c.A124G:p.T42A \\
\hline 11 & 43490831 & C1qtnf2 & $\mathrm{C} 1 \mathrm{q}$ and tumor necrosis factor related protein 2 & c.G379A:p.G127R \\
\hline 11 & 49294204 & Olfr1392 & Olfactory receptor protein 1392 & c.A882T:p.K294N \\
\hline 11 & 50833599 & Zfp879 & Zinc finger protein 879 & c.G410T:p.G137V \\
\hline 11 & 51007734 & Olfr51 & Olfactory receptor protein 51 & c.C761T:p.A254V \\
\hline 11 & 51027442 & Olfr54 & Olfactory receptor protein 54 & c.G439T:p.A147S \\
\hline 11 & 52144841 & Olfr1373 & Olfactory receptor protein 1373 & c.G688A:p.V230M \\
\hline 11 & 107179035 & Nol11 & Nucleolar protein 11 & c.C988T:p.P330S \\
\hline 13 & 21423407 & Pgbd1 & PiggyBac transposable element derived 1 & c.C505T:p.Q169stop \\
\hline 16 & 32753901 & Muc4 & Mucin 4 , cell surface associated & c.C3776A:p.T1259N \\
\hline 17 & 46555608 & Srf & Serum response factor & c.G221T:p.G74V \\
\hline 17 & 73535535 & Galnt14 & UDP-N-acetyl-alpha-D-galactosamine:polypeptide $\mathrm{N}$-acetylgalactosaminyltransferase 14 & c.C519A:p.N173K \\
\hline 18 & 67402164 & Tubb6 & Tubulin, beta 6 class & c.T1132G:p.F378V \\
\hline $\mathrm{x}$ & 20853392 & Araf & v-raf murine sarcoma 3611 viral oncogene homolog & c.G632A:p.R211H \\
\hline $\mathrm{x}$ & 51130161 & Mbn/3 & Muscleblind-like 3 (Drosophila) & c.C715A:p.Q239K \\
\hline
\end{tabular}

doi:10.1371/journal.pone.0127828.t001 


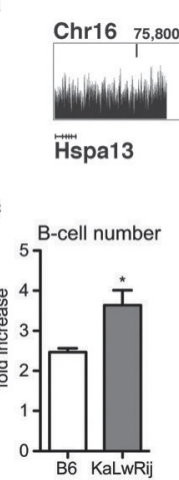

Fig 2. Samsn1 is deleted in KaLwRij and was a negative regulator in B-cells and transformed myeloma cells. (a) Genome sequence coverage is shown for the Samsn1 locus. Physical distance shown along the $x$-axis and the size and location of the region are indicated. Height of the curve represents accumulated sequencing reads. There are virtually no reads across Samsn1 indicating a total deletion of this gene. Hspa13 gene 5' of Samsn1 (shown and labeled) is not affected, and neither are 3' genes AK146213 (shown and labeled) or 4930578N18Rik (first two exons shown but not labeled). Samsn1 is encoded in reverse orientation. (b) Western blot analysis of SAMSN1 in CD43- splenic B-cells from C57BL/ 6 or KaLwRij mice stimulated with IL-4 and LPS for 72 hours. (c) CD43- splenic B-cells were stimulated with IL-4 and LPS for 72 hours and cell number counted pre- and post-stimulation to determine proliferation. (d) Mice were immunized (arrowheads indicate primary and secondary immunization) and serial serum samples collected. IgG2b levels were determined by ELISA. (e) Western blot for Samsn1 in parental 5TGM1 cells, control vector cells (5TGM1-vector), and cells overexpressing Samsn1 (5TGM1-SAMSN1). (f) $5 T G M 1-v e c t o r$ and 5TGM1-SAMSN1 cells were stimulated with IL-6 for 24 hours. Cell proliferation was measured by BrdU incorporation.

doi:10.1371/journal.pone.0127828.g002

(2.48 fold), and Cxcl2 (3.45 fold) expression, transcriptional markers for pro-tumor M2 macrophage polarization. Notably, there were no variants identified in these genes in the KaLwRij genome. Chi3l3 expression was also significantly increased in macrophages isolated from Samsn $1^{-1-}$ mice, confirming that these macrophage expression changes were due specifically to Samsn1 deficiency (Fig 3C).

To test the function of Samsn1 in tumor-associated macrophages in vivo, we injected either wild-type or Samsn1 $1^{-/-}$ex vivo polarized M2 macrophages into established 5TGM1 tumors. Compared to wild-type, Samsn1 $1^{-1-}$ M2 macrophages significantly increased the growth of myeloma tumors (Fig 3D). These findings indicate that Samsn1 regulates a pro-tumor function of macrophages in the myeloma microenvironment.

Interestingly, microarray analysis of Samsn1-nonexpressing BMSCs from C57Bl/6 and KaLwRij also showed many gene expression differences (Fig 4A). Tnfrsf22, Tnfrsf23, and Tnfrsf26, identified in the WGS (S5 Table), showed lower expression in KaLwRij BMSCs compared to C57BL/6 (Fig 4B, S8 Table). Consistent with other reports, expression of Adipoq, previously implicated in MM biology in mice and patients [12], was also significantly reduced in BMSCs from KaLwRij (Fig 4C). Fstl4, initially identified by our integrative genetics approach, was also depleted at steady-state levels in BMSCs (Fig 4D).

\section{Discussion}

High throughput WGS analyses of human MM cell genomes have revealed striking genetic diversity but have failed to clarify the etiology of MM [13, 14]. The spontaneously-derived KaLwRij inbred mouse strain is commonly used as a model for MM but the KaLwRij genome has not previously been sequenced. Here, we present the WGS of the KaLwRij mouse as well as provide gene expression profiles for MM-supportive macrophages and BMSCs as evidence for 
a

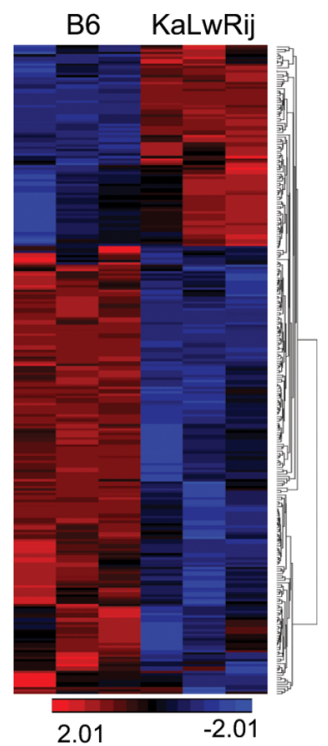

b
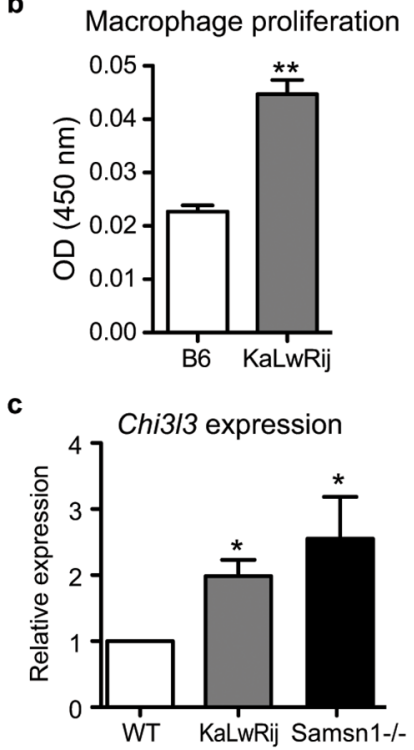

d

5TGM1 tumor volume

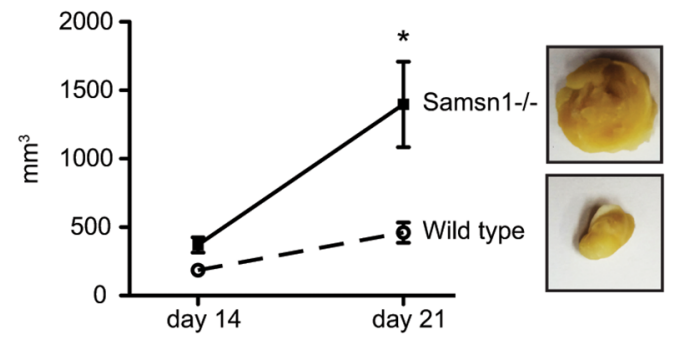

Fig 3. Loss of Samsn1 enhanced pro-tumor macrophage function. (a) Microarray analysis of gene expression in C57BL/6 and KaLwRij primary bone marrow macrophages. (b) Proliferation of C57BL/6 and KaLwRij macrophages was measured by MTT assay. (c) Macrophage M2 polarization marker Chi3l3 in bone marrow macrophages. (d) Wild-type or Samsn $1^{-/-} \mathrm{M} 2$ macrophages polarized ex vivo were injected directly into established 5TGM1 tumors and tumor burden was monitored by bidirectional caliper measurement.

doi:10.1371/journal.pone.0127828.g003

multiple cell lineages contributing to MM prior to overt disease manifestation. Rather than being confined to the B-lineage, the presumed cell of origin of MM, our results suggest that genetic susceptibility alleles are expressed in both the pre-malignant B-cell and in supportive host microenvironment cells.

Combined analysis of loci that contribute to inherited disease risk to both MM in humans and BIP in mice identified Samsn1 as a likely candidate to influence disease susceptibility. Surprisingly, we discovered that Samsn1 is homozygously deleted in the KaLwRij genome [15]. KaLwRij mice have a similar phenotype to Samsn 1-/- mice including increased proliferation of B-cells in ex vivo culture and elevated IgG levels with age [9], supporting the conclusion that absence of Samsn1 drives KaLwRij BIP. Samsn1 add-back inhibited proliferation in transformed MM cells suggesting that the gene can play a plasma-cell intrinsic tumor suppressor role in KaLwRij mice, but we also demonstrated that the absence of Samsn1 in macrophages also contributes to MM progression, making the role of this pathway more complex.

Most compelling were the non-plasma cell contributions of Samsn1 to MM. The majority of genetic alterations in MM cells are already present in MGUS plasma cells [6], suggesting that plasma cell extrinsic factors contribute to the conversion of MGUS to MM. Rather than being 
a

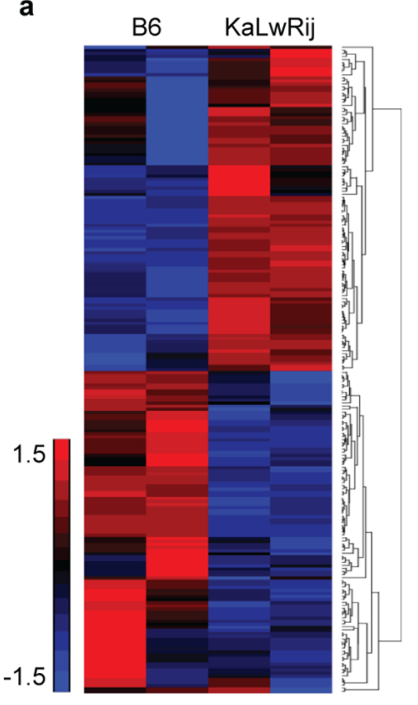

b

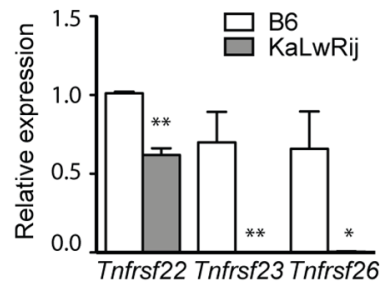

C

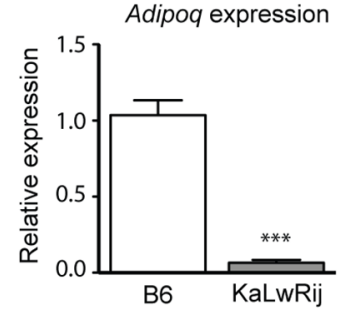

d

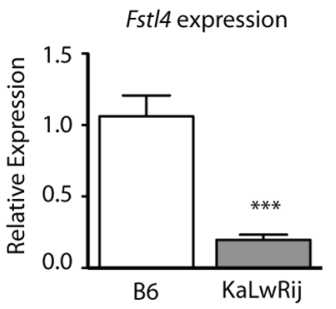

Fig 4. KaLwRij bone marrow stromal cells (BMSCs) have altered gene expression profiles independent of Samsn1. (a) Microarray analysis of B6 and KaLwRij primary BMSCs. (b-d) RT-qPCR analysis of Tnfrsf22, Tnfrsf23, Tnfrsf26, Adipoq, and Fst/4 mRNA levels in B6 and KaLwRij BMSCs. ${ }^{*} \mathrm{P}>0.05,{ }^{*} \mathrm{P}>0.005$, ${ }^{* *} \mathrm{P}<0.0005$.

doi:10.1371/journal.pone.0127828.g004

confined to the affected plasma cell, the genetic susceptibility alleles in KaLwRij also are expressed in supportive host microenvironment cells. Samsn1-null macrophages had increased M2 macrophage markers and potently increased myeloma tumor growth in vivo. These data place Samsn1 as an inhibitor of pro-tumorigenic macrophage polarization and as a plasma-cell extrinsic regulator of MM growth.

Our findings also strongly suggest that gene loci in addition to Samsn1 are likely to be important to MM pathogenesis in the KaLwRij model. We found significant gene expression differences between C57BL/6 and KaLwRij BMSCs, a myeloma-supportive cell type in the tumor microenvironment that does not express Samsn1 (Fig 4). Through WGS, we identified additional KaLwRij genes that may explain differences between KaLwRij and C57BL/6 BMSCs. Notably, we identified deleterious variation and decreased expression in multiple BMSCexpressed genes, including Tnfrsf22, Tnfrsf23, and Tnfrsf26. These genes encode decoy receptors for the TRAIL cytokine [16]. TRAIL-OPG signaling has been implicated in MM progression in humans [17], and these KaLwRij variants may support MM via paracrine signaling.

WGS also identified a novel variant in a paralog of adiponectin (Adipoq), C1qtnf2, predicted to be disruptive. Clqtnf2 is a ubiquitously expressed lipokine with similar function to adiponectin. Adipoq has been shown to have anti-myeloma effects in KaLwRij mice and in humans [12], and we confirmed significantly lower Adipoq expression in KaLwRij BMSCs. Together, these results provide additional lines of evidence that multiple pathways and cell types are 
involved in KaLwRij MM predisposition, and validate our combined genetics approach as a method for identifying pathways involved in MM pathogenesis.

SAMSN1 expression is decreased in MGUS and MM patient samples (S3 Fig) and has reduced expression in human MM cell lines [11], but SAMSN1 is not the target of somatic mutation in human MM [14]. Further work to map the SAMSN1 pathway in humans is needed before we can determine the significance of SAMSN1 to human MM. While SAMSN1 is conserved in humans, one of its murine binding partners, PIRB is not [18]. SAMSN1 is also reported to bind cortactin, a component of integrin signaling and cell migration that is conserved in humans [19], but it is not known whether this interaction is necessary for SAMSN1's role in MM. We pursued investigation of the role of Samsn1 in KaLwRij mouse BIP susceptibility on the basis of a SNP in the human SAMSN1 locus identified in a MM patient GWAS (Fig 2). While SAMSN1 is not deleted in human MM, components of a SAMSN1 pathway appear likely to involved in myeloma susceptibility and/or disease progression.

Our observation that the susceptible background of KaLwRij mice involves multiple cell types helps explain two persistent dilemmas in the myeloma field: i) the marked clonal heterogeneity observed in human MM samples and ii) the inability of human MM cells to engraft in immunocompromised mice without co-transplantation of myeloid and stromal cells [7]. Drug resistance and MM cell survival is well-known to involve several stroma cell types, but the assumption in the field has been that it is the malignant MM cells that subvert normal bone marrow cells to create a pro-tumor milieu. Our findings suggest for the first time that multiple cell lineages are involved in MM pathogenesis prior to disease manifestation and independently of tumor cell somatic mutations. Further evaluation of the interaction between somatic and germline genetic events in the KaLwRij model system may provide additional insights into human MM.

\section{Materials and Methods}

\section{Ethics statement}

The study cohort was approved by the Human Research Protection Office at Washington University School of Medicine and at the Mayo Clinic. Informed written consent from the patients was obtained in accordance with the Declaration of Helsinki. Mice were housed in shared pathogen-free conditions according the guidelines of the Division of Comparative Medicine, Washington University School of Medicine. The Washington University Animal Studies Committee approved all experiments.

\section{Mice}

129S1/SvImJ, A/J, AKR/J, BALB/cByJ, CBA/J, C3H/HeJ, C57BL/6, DBA/2J, FVB/NJ, NOD/ ShiLtJ, SJL/J, and NOD-scid-IL2R $\gamma$ mice were purchased from The Jackson Laboratory. C57Bl/KaLwRij mice were originally obtained from Gregory Mundy at Vanderbilt University. Mice were housed in shared pathogen-free conditions according the guidelines of the Division of Comparative Medicine, Washington University School of Medicine. Mice were euthanized by asphyxiation using $\mathrm{CO}_{2}$ chambers. The animal ethics committee approved all experiments.

Samsn $1^{-/-}$mice were originally obtained from Dingyan Wang [9]. Samsn $1^{-1-}$ and Samsn $1^{+/+}$ mice were housed in shared pathogen-free conditions according to the guidelines from the Animal Resource Centre of the University Health Network, Princess Margaret Hospital Animal Facility. 


\section{Primary cell culture}

Splenic B-cells were negatively selected via MACS with anti-CD43 beads (Miltenyi Biotec). Cells were cultured in RPMI 1650 media, 10\% FBS, 0.00035\% BME, 1\% penicillin-streptomycin and stimulated with $10 \mathrm{ng} / \mathrm{ml}$ LPS and $20 \mathrm{ng} / \mathrm{ml} \mathrm{IL}-4$ for 72 hours. Cells were characterized by FACS (CD43- / B220+ / IgD+, S5 Fig). To generate bone marrow macrophages (BMMs), whole bone marrow was cultured in $\alpha$ MEM, 10\% FBS, $1 \%$ penicillin-streptomycin, $50 \mathrm{ng} / \mathrm{ml} \mathrm{MCSF}$ for 3 days. Cells were characterized by FACS (GR1- / F4/80+, S7 Fig). Proliferation was measured by standard MTT assay (Sigma-Aldrich). M2 polarized macrophages were generated by stimulating BMMs with $5 \mathrm{ng} / \mathrm{ml} \mathrm{IL}-4$ for 24 hours. Bone marrow stromal cells (BMSCs) were generated by plating whole bone marrow cells in ascorbic acid-free aMEM, $10 \%$ FBS, $1 \%$ penicillin-streptomycin for 7 days in $5 \%$ oxygen followed by negative selection via MACS with anti-CD45 beads (Miltenyi Biotec). Cells were characterized by FACS (CD45-, S6 Fig). Flow Cytometric Analysis was performed using FACSCALIBUR (BD Biosciences) and analyzed with FlowJo software (Tree Star). Antibodies used: APC-CD45 (BD Pharmingen), FITC-Gr1 (eBioscience), APC-F4/80 (BioLegend), FITC-CD43 (BD Pharmingen), APC-B220 (eBioscience), and PE-IgD (eBioscience).

\section{TGM1-GFP cell culture}

The 5TGM1-GFP (5TGM1) cell line was originally obtained from Gregory Mundy at Vanderbilt University. Samsn1 cDNA was subconed into an MSCV-PGK-Puro plasmid.

MSCV-PGK-Puro plasmid and lentiviral vectors $\mathrm{pCMV} \Delta 8.9$ and $\mathrm{pM} 2 \mathrm{G}$ were transfected into HEK293T cells. Cell supernatant containing lentivirus was then plated on 5TGM1 cells and selected with puromycin for 72 hours. Cells were maintained in DMEM, 10\% FBS, 1\% penicillinstreptomycin. Proliferation was measured by BrdU ELISA (Roche Diagnostics).

\section{Immunoblotting}

Antibodies: SAMSN1 (Sigma-Aldrich), actin (Sigma-Aldrich), and GFP (Santa Cruz Biotechnology). Blots were incubated with horseradish conjugated secondary antibodies (GE Healthcare) and visualized by chemiluminescence (Pierce Biotechnology).

\section{TGM1 in vivo tumors}

$1 \times 10^{6} 5$ TGM1 cells were injected subcutaneously into the right flank of NOD-scid-IL2R $\gamma$ female mice. 14 days following tumor inoculation, $0.8 \times 10^{6}$ ex vivo M2 polarized macrophages were injected directly into the tumor. Tumor volume was monitored by bidirectional precision caliper measurements $\left(1 / 2 \mathrm{x}\right.$ length $\mathrm{x}$ width $\left.{ }^{2}\right)$. Maximum tumor volume was approximately $2 \mathrm{~cm}^{3}$.

\section{Gene expression microarrays}

RNA was extracted from C57BL/6 and KaLwRij BMMs (3 mice/strain) and BMSCs (2 mice/ strain) using RNeasy Mini kit (Qiagen). RNA samples were submitted to the Genome Technology Access Center at Washington University School of Medicine for hybridization using the GeneChip Mouse Gene 1.0 ST array (Affymetrix). Differentially expressed genes were defined as $\geq 1.5$ fold changed between C57BL/ 6 and KaLwRij.

We re-analyzed the microarray data of CD138+ plasma cells from healthy donors, MGUS patients, and MM patients published by Fonseca, R. et al. (GSE6477) [20, 21].

Data was analyzed using Partek Genomics Suite (Partek Inc.). 


\section{Quantitative reverse transcription PCR}

RNA was extracted using RNeasy Mini kit (Qiagen) and cDNA generated using iScript (BioRad). Quantitative PCR was completed using SsoFast EVA Green Supermix or iQ Supermix (Bio-Rad). All samples run with biological replicates of $\geq 2$. Primer sequences are in S1 Methods.

\section{Statistics}

Data are shown as mean +/- SEM. Unless otherwise indicated, experiments were analyzed using Student's t-test to compare 2 groups or ANOVA to compare multiple groups. ${ }^{*} \mathrm{p}<0.05$; ${ }^{* *} \mathrm{p}<0.01 ;{ }^{* * *} \mathrm{p}<0.001$.

\section{Supporting Information}

S1 Fig. Immune responses are significantly different among mouse strains. Schema for immunization and serial serum sample protocol. Serum was collected at baseline (T0-5 months), post-primary immunization (T1-7 months), post-boosting immunization (T2-8.5 months), 12 months (T3), and 18 months (T4). Analysis of serial serum samples by ELISA for (b) immunoglobulin isotype $G$, (c) immunoglobulin isotype $M$, and (d) immunoglobulin isoype A. (e) Representative SPEP of mouse serum samples negative (-) and positive (+) for M-spike. (PDF)

S2 Fig. Samsn1 is deleted in KaLwRij and expression varies by cell type in C57BL/6. (a) PCR amplification of the regions surrounding the 180kb deletion including Samsn1 identified via WGS of the KaLwRij strain. Primers flanking the breakpoint amplified a product in KaLwRij genomic DNA and KaLwRij-derived 5TGM1 myeloma cell line DNA, but not C57BL/6 genomic DNA. (b) Samsn1 mRNA expression was measured by RT-qPCR in multiple cell types. CD43- B cells were analyzed pre- and post-stimulation for 72hrs with IL4 and LPS. Macrophages were analyzed pre- and post-polarization to a M2 phenotype using IL4. CD45BMSCs were also analyzed for Samsn1 expression. ${ }^{* *} \mathrm{P}<0.005$, *** $\mathrm{P}<0.0005$. (PDF)

S3 Fig. SAMSN1 is expressed at a lower level in human MGUS and MM plasma cells. Microarray data from CD138+ plasma cells from human healthy donors, MGUS patients, and MM patients, first published by R. Fonseca et al. in 2006 (GEO accession: GSE6477) was analyzed for SAMSN1 expression levels. ${ }^{*} P<0.05,{ }^{* * *} P>0.0001$.

(PDF)

S4 Fig. FACS analysis of splenic B-cells. Isolated mouse splenocytes were negatively selected by magnetic immunodepletion, using anti-CD43 beads (Miltenyi Biotec). The negative fraction was then analysed by FACS. The $\mathrm{CD}_{4} 3^{-}$(middle panel) and naive $\mathrm{B}$-cell $\left(\mathrm{B} 220^{+}\right.$, $\mathrm{IgD}^{+}$, right panel) populations are shown.

(PDF)

S5 Fig. FACS analysis of bone marrow stromal cells. Whole bone marrow cells were cultured ascorbic acid-free $\alpha \mathrm{MEM}, 10 \%$ FBS, $1 \%$ penicillin-streptomycin for 7 days in $5 \%$ oxygen (left panels, "pre-sorted"). On day 7, cells were negatively selected by MACS with anti-CD45 beads (right panels).

(PDF)

S6 Fig. FACS analysis of bone marrow macrophages. Whole bone marrow was cultured in $\alpha \mathrm{MEM}, 10 \% \mathrm{FBS}, 1 \%$ penicillin-streptomycin, $50 \mathrm{ng} / \mathrm{ml}$ MCSF for 3 days. After 3 days, the cell 
population is enriched for GR1- / F4/80+ macrophages.

(PDF)

S7 Fig. FACS analysis of bone marrow macrophages. Whole bone marrow was cultured in $\alpha$ MEM, 10\% FBS, $1 \%$ penicillin-streptomycin, $50 \mathrm{ng} / \mathrm{ml} \mathrm{MCSF}$ for 3 days. After 3 days, the cell population is enriched for GR1- / F4/80+ macrophages.

S1 Methods. Supplemental section with expanded details regarding the experimental methods used in this manuscript.

(DOCX)

S1 Table. Mice with positive M-spike on serum protein electrophoresis. (XLSX)

S2 Table. Candidate genes underlying genetic susceptibility to BIP in KaLwRij mice. (XLSX)

S3 Table. Candidate genes underlying genetic susceptibility to multiple myeloma in humans.

(XLSX)

S4 Table. KaLwRij germline structural variants.

(XLSX)

S5 Table. KaLwRij germline SNVs.

(XLSX)

S6 Table. KaLwRij germline Indels.

(XLSX)

S7 Table. Frameshift and splice site mutations in the KaLwRij germline.

(DOCX)

S8 Table. Microarray analysis of BMM and BMSC gene expression. (XLSX)

\section{Author Contributions}

Conceived and designed the experiments: SRA WCW LC XS KNW MHT. Performed the experiments: SRA WCW LC LL XS YX MH JO. Analyzed the data: SRA WCW PL DS CMV MH. Contributed reagents/materials/analysis tools: DW AG CMV GC RV XYW. Wrote the paper: SRA WCW KNW MHT JO.

\section{References}

1. Kumar SK, Dispenzieri A, Lacy MQ, Gertz MA, Buadi FK, Pandey S, et al. Continued improvement in survival in multiple myeloma: changes in early mortality and outcomes in older patients. Leukemia. 2013.

2. Landgren O, Gridley G, Turesson I, Caporaso NE, Goldin LR, Baris D, et al. Risk of monoclonal gammopathy of undetermined significance (MGUS) and subsequent multiple myeloma among African American and white veterans in the United States. Blood. 2006; 107(3):904-6. PMID: 16210333

3. Kyle RA, Durie BG, Rajkumar SV, Landgren O, Blade J, Merlini G, et al. Monoclonal gammopathy of undetermined significance (MGUS) and smoldering (asymptomatic) multiple myeloma: IMWG consensus perspectives risk factors for progression and guidelines for monitoring and management. Leukemia. 2010; 24(6):1121-7. doi: 10.1038/leu.2010.60 PMID: 20410922 
4. Kyle RA, Therneau TM, Rajkumar SV, Larson DR, Plevak MF, Offord JR, et al. Prevalence of monoclonal gammopathy of undetermined significance. The New England journal of medicine. 2006; 354 (13):1362-9. PMID: 16571879

5. Weinhold N, Johnson DC, Rawstron AC, Forsti A, Doughty C, Vijayakrishnan J, et al. Inherited genetic susceptibility to monoclonal gammopathy of unknown significance. Blood. 2014; 123(16):2513-7. doi: 10.1182/blood-2013-10-532283 PMID: 24449210

6. Davies FE, Dring AM, Li C, Rawstron AC, Shammas MA, O'Connor SM, et al. Insights into the multistep transformation of MGUS to myeloma using microarray expression analysis. Blood. 2003; 102 (13):4504-11. PMID: 12947006

7. Epstein J, Yaccoby S. The SCID-hu myeloma model. (1543-1894 (Print)).

8. Radl J, Hollander CF, van den Berg P, de Glopper E. Idiopathic paraproteinaemia. I. Studies in an animal model-the ageing C57BL/KaLwRij mouse. Clinical and experimental immunology. 1978; 33 (3):395-402. PMID: 367647

9. Wang D, Stewart AK, Zhuang L, Zhu Y, Wang Y, Shi C, et al. Enhanced adaptive immunity in mice lacking the immunoinhibitory adaptor Hacs1. FASEB journal: official publication of the Federation of American Societies for Experimental Biology. 2010; 24(3):947-56.

10. Garrett IR, Dallas S, Radl J, Mundy GR. A murine model of human myeloma bone disease. Bone. 1997; 20(6):515-20. PMID: 9177864

11. Claudio JO, Zhu YX, Benn SJ, Shukla AH, McGlade CJ, Falcioni N, et al. HACS1 encodes a novel SH3-SAM adaptor protein differentially expressed in normal and malignant hematopoietic cells. Oncogene. 2001; 20(38):5373-7. PMID: 11536050

12. Fowler JA, Lwin ST, Drake MT, Edwards JR, Kyle RA, Mundy GR, et al. Host-derived adiponectin is tumor-suppressive and a novel therapeutic target for multiple myeloma and the associated bone disease. Blood. 2011; 118(22):5872-82. doi: 10.1182/blood-2011-01-330407 PMID: 21908434

13. Lohr JG, Stojanov P, Carter SL, Cruz-Gordillo P, Lawrence MS, Auclair D, et al. Widespread genetic heterogeneity in multiple myeloma: implications for targeted therapy. (1878-3686 (Electronic)).

14. Chapman MA, Lawrence MS, Keats JJ, Cibulskis K, Sougnez C, Schinzel AC, et al. Initial genome sequencing and analysis of multiple myeloma. Nature. 2011; 471(7339):467-72. doi: 10.1038/ nature09837 PMID: 21430775

15. Sarah Amend LC, Daniel Serie, Celine M Vachon, Lan Lu, Ravi Vij, Graham A Colditz, Katherine N Weilbaecher, Michael H. Tomasson, editor Deletion of samsn1 underlies genetic susceptibility to MGUS in mice. 55th American Society of Hematology Annual Meeting and Exposition; Dec. 9th, 2013; New Orleans, LA: 55th American Society of Hematology Annual Meeting and Exposition.

16. Schneider P, Olson D Fau-Tardivel A, Tardivel A Fau—Browning B, Browning B Fau—Lugovskoy A, Lugovskoy A Fau-Gong D, Gong D Fau—Dobles M, et al. Identification of a new murine tumor necrosis factor receptor locus that contains two novel murine receptors for tumor necrosis factor-related apoptosis-inducing ligand (TRAIL). (0021-9258 (Print)).

17. Colucci S, Brunetti G, Rizzi R, Zonno A, Mori G, Colaianni G, et al. T cells support osteoclastogenesis in an in vitro model derived from human multiple myeloma bone disease: the role of the OPG/TRAIL interaction. Blood. 2004; 104(12):3722-30. PMID: 15308561

18. Zhu YX, Benn S, Li ZH, Wei E, Masih-Khan E, Trieu Y, et al. The SH3-SAM adaptor HACS1 is up-regulated in B cell activation signaling cascades. The Journal of experimental medicine. 2004; 200(6):73747. PMID: 15381729

19. von Holleben M, Gohla A, Janssen KP, Iritani BM, Beer-Hammer S. Immunoinhibitory adapter protein Src homology domain 3 lymphocyte protein 2 (SLy2) regulates actin dynamics and B cell spreading. The Journal of biological chemistry. 2011; 286(15):13489-501. doi: 10.1074/jbc.M110.155184 PMID: 21296879

20. Chng WJ, Kumar S, Vanwier S, Ahmann G, Price-Troska T, Henderson K, et al. Molecular dissection of hyperdiploid multiple myeloma by gene expression profiling. Cancer research. 2007; 67(7):2982-9. PMID: 17409404

21. Edgar R, Domrachev M, Lash AE. Gene Expression Omnibus: NCBI gene expression and hybridization array data repository. Nucleic acids research. 2002; 30(1):207-10. doi: D-NLM: PMC99122 EDAT2001/12/26 10:00 MHDA- 2002/01/22 10:01 CRDT-2001/12/26 10:00 PST—ppublish. PMID: 11752295 